\title{
BMJ Open Hospitalisation with community- acquired pneumonia among patients with type 2 diabetes: an observational population-based study in Spain from 2004 to 2013
}

Ana López-de-Andrés, ${ }^{1}$ Javier de Miguel-Díez, ${ }^{2}$ Isabel Jiménez-Trujillo, ${ }^{1}$ Valentín Hernández-Barrera, ${ }^{1}$ José M de Miguel-Yanes, ${ }^{3}$ Manuel Méndez-Bailón, ${ }^{4}$ Napoleón Pérez-Farinós, ${ }^{5}$ Miguel Á ngel Salinero-Fort, ${ }^{6}$ Rodrigo Jiménez-García ${ }^{1}$

To cite: López-de-Andrés A, de Miguel-Díez J, JiménezTrujillo I, et al. Hospitalisation with community-acquired pneumonia among patients with type 2 diabetes: an observational populationbased study in Spain from 2004 to 2013. BMJ Open 2017;7:e013097. doi:10.1136/bmjopen-2016013097

- Prepublication history for this paper is available online. To view these files please visit the journal online (http://dx.doi.org/10.1136/ bmjopen-2016-013097).

Received 20 June 2016 Revised 10 October 2016 Accepted 27 October 2016

CrossMark

For numbered affiliations see end of article.

Correspondence to Dr Ana López de Andrés; ana.lopez@urjc.es

\section{ABSTRACT}

Objectives: To describe trends in the incidence and outcomes of community-acquired pneumonia (CAP) hospitalisations among patients with or without diabetes in Spain (2004-2013).

Design: Retrospective, observational study using the Spanish National Hospital Discharge Database (Conjunto Mínimo Básico de Datos (CMBD)).

Setting: Spain.

Participants: We used national hospital discharge data to select all hospital admissions for CAP.

Main outcome measures: Incidence was calculated overall and stratified by diabetes status: type 2 diabetes mellitus (T2DM) and no diabetes.

Results: We identified 901136 admissions for CAP (24.8\% with T2DM). Incidence rates of CAP increased significantly in patients with T2DM over time. The incidence was higher among people with T2DM for all time periods. Patients with T2DM were older and had higher comorbidity index than non-diabetics.

Streptococcus pneumoniae decreased over time for both groups. Time trend analyses showed significant decreases in mortality during admission for CAP for patients with and without T2DM. Factors associated with higher mortality in both groups included: older age, higher comorbidity, mechanical ventilation, red cell transfusion, readmission and Staphylococcus aureus detection. Diabetes was associated with a lower in-hospital mortality (OR $0.92,95 \% \mathrm{Cl} 0.91$ to 0.94 ) after a CAP hospitalisation.

Conclusions: CAP incidence rates were higher and increased over time at a higher rate among patients with T2DM. Mortality decreased over time in all groups. The presence of diabetes is not a risk factor for death during admission for CAP.

\section{INTRODUCTION}

Prevalence of diabetes is steadily rising. In Spain, the number of people with diabetes

\section{Strengths and limitations of this study}

- The strengths of our findings lie in the large sample size, the 10-year follow-up period, and the standardised methodology.

- Our findings are limited by the lack of data, precluding adjustment for pneumococcal and influenza vaccinations, which have been associated with reduced mortality among patients hospitalised with pneumonia.

- We have not identified factors (specimen quality or antimicrobial treatments) that may influence community-acquired pneumonia outcomes because these variables were not collected in the Spanish Hospital Discharge Database.

- We did not classify diabetic patients into groups based on the therapy used to control blood glucose, with the result that we were unable to provide data on the control of blood glucose during the hospitalisation.

has more than doubled over the last decade due to an increasing obesity rate and an ageing population. ${ }^{1}$ This increase in diabetes prevalence is projected to lead a significant increase in patients with community-acquired pneumonia (CAP). ${ }^{2}$

CAP is a leading infectious cause of hospitalisation worldwide, particularly among people with diabetes. $^{3-5}$ Previous studies have shown that diabetes is a risk factor for a pneumoniarelated hospitalisation. ${ }^{6-8}$ A population-based cohort study found that the adjusted relative risk (RR) for pneumonia-related hospitalisation among subjects with diabetes was 1.26 (95\% CI 1.21 to 1.31 ) compared with nondiabetic patients. ${ }^{4}$

Advanced age and comorbidity are associated with increased mortality among adults hospitalised with CAP. ${ }^{9}$ Diabetic patients may 
have increased susceptibility to pneumonia for several reasons. They are at increased risk of hyperglycaemia, decreased immunity, impaired lung function and chronic complications such as heart disease, renal failure and pulmonary microangiopathy. ${ }^{10}$ Kornum et al concluded that presence of type 2 diabetes mellitus (T2DM) predicts increased pneumonia-related mortality. However, Kaplan et $a l^{11}$ reported no association between in-hospital mortality (IHM) and diabetes.

The incidence of pneumonia may be increasing. ${ }^{3} 912$ Secular trends in incidence and outcomes of CAP among patients with and without T2DM have been examined. ${ }^{4-6}$ However, to the best of our knowledge, no previous studies have investigated national trends in the incidence, characteristics and outcomes of CAP in people with diabetes in Spain.

In this study, we used national hospital discharge data to examine trends in incidence and outcomes of CAP among patients with or without T2DM in Spain from 2004 to 2013. In particular, we analysed patient comorbidities, diagnostic and therapeutic procedures, pneumonia pathogens and in-hospital outcomes, such as readmission, IHM and length of hospital stay (LOHS).

\section{METHODS}

We performed a retrospective, observational study using the Spanish National Hospital Discharge Database (Conjunto Mínimo Básico de Datos (CMBD)), which compiles all public and private hospital data, covering more than $98 \%$ of hospital admissions. ${ }^{13}$ The CMBD includes patient variables (sex, date of birth), admission and discharge dates, up to 14 discharge diagnoses, and up to 20 procedures performed during the hospital stay. ${ }^{13}$ We analysed data collected between 1 January 2004 and 31 December 2013 for subjects aged 40 and over.

The criteria for diseases and procedures were defined according to the International Classification of Diseases, Ninth Revision, Clinical Modification (ICD-9-CM), which is used in the Spanish CMBD.

We selected admissions for patients with a primary diagnosis of CAP (ICD-9-CM codes: 480-488, 507.0507.8). We grouped admissions by diabetes status as follows: T2DM (ICD-9-CM codes: 250.x0 and 250.x2) or no diabetes in any diagnostic position. We excluded people with type 1 diabetes mellitus (ICD-9-CM codes: 250.x1; 250.x3).

Clinical characteristics included information on overall comorbidity at the time of diagnosis, which was assessed by calculating the Charlson Comorbidity Index (CCI) ${ }^{14}$ We divided patients into three categories: low index, which corresponds to patients with no previously recorded disease; medium index, patients with one disease category; and high index, patients with two or more disease categories.

Irrespective of the position at the diagnoses coding list, we retrieved data about comorbidities as described by Kornum et al (2007). ${ }^{5}$ Also, we specifically identified the following procedures: computerised axial tomography (CAT) of thorax (ICD-9-CM code 87.41), bronchial fibroscopy (ICD-9-CM code 33.21-33.24), noninvasive mechanical ventilation (ICD-9-CM code 93.90), invasive mechanical ventilation (ICD-9-CM code 96.7, 96.70, 96.71, 96.72), thoracocentesis (ICD-9-CM code 34.91) and red cell transfusion (ICD-9-CM code 99.03, 99.04).

We analysed pneumonia pathogens documented during hospitalisations for pneumonia using the following ICD-9-CM codes: 481 for Streptococcus pneumoniae; 482.84 for Legionella; 482.41 and 482.42 for Staphylococcus aureus; 482.2 for Haemophilus influenzae; and 482.1 for Pseudomonas aeruginosa. These were the five most frequently identified pathogens. All others represented under $0.30 \%$ of admissions.

We estimated the proportion of readmission (patients that had been discharged from the hospital within the previous 30 days), the median of LOHS and IHM. IHM is defined by the proportion of patients who died during admission for each year of study.

\section{Statistical analysis}

In order to assess time trends, the age and sex incidence rates of admissions for CAP in patients with T2DM and non-diabetic patients were calculated per 100000 inhabitants,. We calculated yearly T2DM-specific incidence rates by dividing the number of admissions per year, sex, and age group by the corresponding number of people in that population group using the age-adjusted, sexadjusted estimated prevalence of T2DM obtained from National Health Surveys (NHS) conducted in 2003/ 2004, 2006/2007, 2009/2010 and 2011/2012, and based on data from the Di@bet.es Study, which estimated the prevalence of diabetes in the Spanish population. ${ }^{1}{ }^{15}$ From 2001 to 2010, Spanish NHS was published every 2 or 3 years. So diabetic population for missing years (2005 and 2008) was estimated assuming that growth rate was the same thorough the period 2004-2010. We estimated rates by fitting a linear regression model with population from years when NHS was available and we used this model to impute population for 2005 and 2008. We also calculated the yearly, age and sex adjustedspecific incidence rates for non-diabetic patients by dividing the number of cases per year, sex, and age group by the corresponding number of people in that population group (excluding those with T2DM), according to the data from the Spanish National Institute of Statistics, as reported on 31 December of each year. ${ }^{16}$

To assess the effect of T2DM on the incidence, we fitted two separate multivariate Poisson regression models for patients with and without T2DM adjusted by sex, age and year of discharge as independent variables. The results of these models are shown as adjusted incidence rate ratio (IRR) with their 95\% CIs. A model adjusting by the same independent variables and including diabetes status was also conducted to assess the 
adjusted effect of diabetes in the incidence of the total population.

To assess whether there was any overinflation, we tried also with models of negative binomial regression, obtaining very similar results so we decided to use conventional Poisson regression models.

A descriptive statistical analysis was performed for all continuous variables and categories by stratifying admissions for CAP according to diabetes status. Variables are expressed as proportions, as means with SDs or as medians with IQRs (LOHS). A bivariate analysis of variables according to year was performed using the $\chi^{2}$ test for linear trend (proportions), analysis of variance (ANOVA) (means) and Kruskal-Wallis (medians), as appropriate.

To assess differences between those patients with and without T2DM, for each year and for the total sample, the statistical tests conducted for continuous variables were the Student's t-test for normal distributions and the Mann-Whitney test for non-normal distributions; categorical variables were compared using the $\chi^{2}$ test and adjusted incidences were compared using Poisson regression. These same tests were used to compare the characteristics of those diabetic patients who died with those who survived to the hospital admission and equally for non-diabetic subjects. Finally, we performed logistic regression analyses with mortality as a binary outcome using the independent variables and age, sex, CCI, readmission, diagnostic and therapeutic procedures, pathogens and year of admission for those with and without diabetes and for the entire population to assess the influence of diabetes on IHM. Estimates were ORs with their 95\% CIs. Statistical analyses were performed using Stata V.10.1 (Stata, College Station, Texas, USA). Statistical significance was set at $\mathrm{p}<0.05$ (two-tailed).

\section{Ethical aspects}

Data confidentiality was maintained at all times in accordance with Spanish legislation. Given the anonymous and mandatory nature of the data set, it was not deemed necessary to obtain informed consent.

\section{RESULTS}

From 2004 to 2013, we identified a total of 901136 admissions for CAP as primary diagnosis in patients aged $\geq 40$ years in Spain. Patients with T2DM accounted for $24.8 \%$ of total (134534 men and 89181 women).

Table 1 and table 2 show the incidence and the clinical characteristics, comorbidities, diagnostic and therapeutic procedures and in-hospital outcomes of admissions for CAP in patients with T2DM and in patients without T2DM from 2004 to 2013, respectively.

Among patients with T2DM, adjusted incidence of admissions for CAP increased significantly from 812.64 cases per 100000 T2DM population in 2004 to 923.26 cases in 2013 (table 1). In patients without T2DM the adjusted incidence of admissions increased significantly from 316.24 cases per 100000 population without diabetes in 2004 to 341.98 in 2013 (table 2). Incidence was significantly higher in people with T2DM than in nondiabetic people for all years analysed. From 2004 to 2013, the adjusted IRR of having CAP admission diagnosis in patients with T2DM was significant and higher than in those without diabetes (IRR 1.27, 95\% CI 1.23 to 1.31 vs IRR $1.05,95 \%$ CI 1.03 to 1.07 ).

Using the Poisson regression model, including the total population and diabetes status as an independent variable, we obtained an adjusted IRR per year of 1.66 (95\% CI 1.65 to 1.67) for patients with T2DM using those without diabetes as the reference category. In other words, the incidence of admissions for CAP over the entire period was 1.66-times higher among patients with T2DM than those without diabetes.

In patients who have an admission for CAP, there was a significant male predominance $(60.14 \%$ for T2DM and $60.95 \%$ for no diabetes). Overall, patients with T2DM were significantly older (77.08; $\mathrm{SD}=10.46$ years) than patients without diabetes (75.06; $\mathrm{SD}=13.76$ years) and had more coexisting medical conditions. Specifically, had higher prevalence of acute myocardial infarction $(4.8 \%$ vs $3.1 \%)$, congestive heart failure ( $18.54 \%$ vs $13.53 \%)$, cerebrovascular disease/hemiplegia/paraplegia ( $10.3 \%$ vs $7.68 \%$ ), dementia ( $11.21 \%$ vs $10.72 \%)$, renal disease $(15.43 \%$ vs $10.09 \%)$, peripheral vascular disease $(6.14 \%$ vs $3.62 \%)$ and prevalence of obesity is two times higher (all $\mathrm{p}<0.05$ ). On the other hand, any type of malignancy and pleuritis were more prevalent in non-diabetic patients $(10.6 \%$ and $7.03 \%$, respectively) than in those with T2DM $(7.94 \%$ and $5.94 \%$, respectively). Age and all these comorbidities increased significantly over time in people with T2DM and without diabetes (table 1 and table 2).

As can been seen in table 1 and table 2, acute myocardial infarction and chronic pulmonary disease decreased significantly in both groups over the study period. Male sex percentage increased significantly in people with T2DM and female percentage showed a much larger change over time in patients without T2DM.

We detected a significant increase in use of thorax CAT in both groups over the study period as can been seen in table 1 and table 2 .

The use of all therapeutics procedures (except invasive mechanical ventilation which showed a significant decrease) have significantly increased in the last 10 years in diabetic and non-diabetic patients (table 1 and table 2). The use of non-invasive mechanical ventilation has shown an over threefold increase in both groups of patients over the study period.

Of the pathogens analysed the most commonly found was $S$ pneumoniae, followed by Legionella, $P$ aeruginosa, $S$ aureus and $H$ influenzae.

In year 2013, S pneumoniae was detected in $7.95 \%$ of diabetic patients and $8.47 \%$ in those without the disease. All other pathogens were found in $<1 \%$ of patients. 
Table 1 Incidence and characteristics of hospital admissions for pneumonia as primary diagnosis in patients with type 2 diabetes in Spain, 2004-2013

\begin{tabular}{|c|c|c|c|c|c|c|c|c|c|c|c|}
\hline & 2004 & 2005 & 2006 & 2007 & 2008 & 2009 & 2010 & 2011 & 2012 & 2013 & Total \\
\hline $\mathrm{N}$ & 16161 & 19764 & 17267 & 20913 & 22002 & 24426 & 23377 & 25807 & 27655 & 26343 & 223715 \\
\hline Incidence* (per 100000 inhabitants) & 812.64 & 948.39 & 792.36 & 959.67 & 974.43 & 1045.33 & 1000.43 & 962.56 & 1031.49 & 923.26 & 948.84 \\
\hline Women, $\mathrm{n}(\%)^{\star}$ & $\begin{array}{l}6476 \\
(40.07)\end{array}$ & $\begin{array}{l}8060 \\
(40.78)\end{array}$ & $\begin{array}{l}6632 \\
(38.41)\end{array}$ & $\begin{array}{l}8364 \\
(39.99)\end{array}$ & $\begin{array}{l}8678 \\
(39.44)\end{array}$ & $\begin{array}{l}9880 \\
(40.45)\end{array}$ & $\begin{array}{l}9240 \\
(39.53)\end{array}$ & $\begin{array}{l}10338 \\
(40.06)\end{array}$ & $\begin{array}{l}11126 \\
(40.23)\end{array}$ & $\begin{array}{l}10387 \\
(39.43)\end{array}$ & $\begin{array}{l}89181 \\
(39.86)\end{array}$ \\
\hline Age, mean (SD) & $\begin{array}{l}75.97 \\
(10.24)\end{array}$ & $\begin{array}{l}76.06 \\
(10.39)\end{array}$ & $\begin{array}{l}76.18 \\
(10.47)\end{array}$ & $\begin{array}{l}76.29 \\
(10.39)\end{array}$ & $\begin{array}{l}76.91 \\
(10.47)\end{array}$ & $\begin{array}{l}76.37 \\
(10.98)\end{array}$ & $\begin{array}{l}77.53 \\
(10.28)\end{array}$ & $\begin{array}{l}77.64 \\
(10.45)\end{array}$ & $\begin{array}{l}78.41 \\
(10.15)\end{array}$ & $\begin{array}{l}78.23 \\
(10.34)\end{array}$ & $\begin{array}{l}77.08 \\
(10.46)\end{array}$ \\
\hline 40-64 years, n (\%)† & $\begin{array}{l}2150 \\
(13.3)\end{array}$ & $\begin{array}{l}2638 \\
(13.35)\end{array}$ & $\begin{array}{l}2348 \\
(13.6)\end{array}$ & $\begin{array}{l}2864 \\
(13.69)\end{array}$ & $\begin{array}{l}2894 \\
(13.15)\end{array}$ & $\begin{array}{l}3589 \\
(14.69)\end{array}$ & $\begin{array}{l}2690 \\
(11.51)\end{array}$ & $\begin{array}{l}3085 \\
(11.95)\end{array}$ & $\begin{array}{l}2892 \\
(10.46)\end{array}$ & $\begin{array}{l}2846 \\
(10.8)\end{array}$ & $\begin{array}{l}27996 \\
(12.51)\end{array}$ \\
\hline 65-74 years, $n(\%) \dagger$ & $\begin{array}{l}4329 \\
(26.79)\end{array}$ & $\begin{array}{l}5096 \\
(25.78)\end{array}$ & $\begin{array}{l}4302 \\
(24.91)\end{array}$ & $\begin{array}{l}5017 \\
(23.99)\end{array}$ & $\begin{array}{l}4780 \\
(21.73)\end{array}$ & $\begin{array}{l}5262 \\
(21.54)\end{array}$ & $\begin{array}{l}4880 \\
(20.88)\end{array}$ & $\begin{array}{l}5178 \\
(20.06)\end{array}$ & $\begin{array}{l}5030 \\
(18.19)\end{array}$ & $\begin{array}{l}5027 \\
(19.08)\end{array}$ & $\begin{array}{l}48901 \\
(21.86)\end{array}$ \\
\hline $75-84$ years, $n(\%)^{*}$ & $\begin{array}{l}6385 \\
(39.51)\end{array}$ & $\begin{array}{l}7925 \\
(40.1)\end{array}$ & $\begin{array}{l}6962 \\
(40.32)\end{array}$ & $\begin{array}{l}8468 \\
(40.49)\end{array}$ & $\begin{array}{l}8972 \\
(40.78)\end{array}$ & $\begin{array}{l}9782 \\
(40.05)\end{array}$ & $\begin{array}{l}9749 \\
(41.7)\end{array}$ & $\begin{array}{l}10407 \\
(40.33)\end{array}$ & $\begin{array}{l}11515 \\
(41.64)\end{array}$ & $\begin{array}{l}10651 \\
(40.43)\end{array}$ & $\begin{array}{l}90816 \\
(40.59)\end{array}$ \\
\hline$\geq 85$ years, $n(\%)^{*}$ & $\begin{array}{l}3297 \\
(20.4)\end{array}$ & $\begin{array}{l}4105 \\
(20.77)\end{array}$ & $\begin{array}{l}3655 \\
(21.17)\end{array}$ & $\begin{array}{l}4564 \\
(21.82)\end{array}$ & $\begin{array}{l}5356 \\
(24.34)\end{array}$ & $\begin{array}{l}5793 \\
(23.72)\end{array}$ & $\begin{array}{l}6058 \\
(25.91)\end{array}$ & $\begin{array}{l}7137 \\
(27.66)\end{array}$ & $\begin{array}{l}8218 \\
(29.72)\end{array}$ & $\begin{array}{l}7819 \\
(29.68)\end{array}$ & $\begin{array}{l}56002 \\
(25.03)\end{array}$ \\
\hline AMI, n (\%)† & $\begin{array}{l}865 \\
(5.35)\end{array}$ & $\begin{array}{l}1106 \\
(5.6)\end{array}$ & $\begin{array}{l}991 \\
(5.74)\end{array}$ & $\begin{array}{l}1180 \\
(5.64)\end{array}$ & $\begin{array}{l}1167 \\
(5.3)\end{array}$ & $\begin{array}{l}1141 \\
(4.67)\end{array}$ & $\begin{array}{l}958 \\
(4.1)\end{array}$ & $\begin{array}{l}1098 \\
(4.25)\end{array}$ & $\begin{array}{l}1184 \\
(4.28)\end{array}$ & $\begin{array}{l}1057 \\
(4.01)\end{array}$ & $\begin{array}{l}10747 \\
(4.8)\end{array}$ \\
\hline $\mathrm{CHF}, \mathrm{n}(\%)^{*}$ & $\begin{array}{l}2587 \\
(16.01)\end{array}$ & $\begin{array}{l}3258 \\
(16.48)\end{array}$ & $\begin{array}{l}2941 \\
(17.03)\end{array}$ & $\begin{array}{l}3542 \\
(16.94)\end{array}$ & $\begin{array}{l}3940 \\
(17.91)\end{array}$ & $\begin{array}{l}4183 \\
(17.13)\end{array}$ & $\begin{array}{l}4379 \\
(18.73)\end{array}$ & $\begin{array}{l}5210 \\
(20.19)\end{array}$ & $\begin{array}{l}5795 \\
(20.95)\end{array}$ & $\begin{array}{l}5645 \\
(21.43)\end{array}$ & $\begin{array}{l}41480 \\
(18.54)\end{array}$ \\
\hline PVD, $\mathrm{n}(\%)^{*}$ & $\begin{array}{l}950 \\
(5.88)\end{array}$ & $\begin{array}{l}1205 \\
(6.1)\end{array}$ & $\begin{array}{l}1041 \\
(6.03)\end{array}$ & $\begin{array}{l}1249 \\
(5.97)\end{array}$ & $\begin{array}{l}1239 \\
(5.63)\end{array}$ & $\begin{array}{l}1391 \\
(5.69)\end{array}$ & $\begin{array}{l}1371 \\
(5.86)\end{array}$ & $\begin{array}{l}1681 \\
(6.51)\end{array}$ & $\begin{array}{l}1728 \\
(6.25)\end{array}$ & $\begin{array}{l}1874 \\
(7.11)\end{array}$ & $\begin{array}{l}13729 \\
(6.14)\end{array}$ \\
\hline CEVD/HP/PAPL, n (\%)* & $\begin{array}{l}1509 \\
(9.34)\end{array}$ & $\begin{array}{l}1850 \\
(9.36)\end{array}$ & $\begin{array}{l}1688 \\
(9.78)\end{array}$ & $\begin{array}{l}1959 \\
(9.37)\end{array}$ & $\begin{array}{l}2290 \\
(10.41)\end{array}$ & $\begin{array}{l}2496 \\
(10.22)\end{array}$ & $\begin{array}{l}2535 \\
(10.84)\end{array}$ & $\begin{array}{l}2753 \\
(10.67)\end{array}$ & $\begin{array}{l}3101 \\
(11.21)\end{array}$ & $\begin{array}{l}2854 \\
(10.83)\end{array}$ & $\begin{array}{l}23035 \\
(10.3)\end{array}$ \\
\hline Chronic pulmonary disease, $\mathrm{n}(\%) \dagger$ & $\begin{array}{l}5618 \\
(34.76)\end{array}$ & $\begin{array}{l}6693 \\
(33.86)\end{array}$ & $\begin{array}{l}5730 \\
(33.18)\end{array}$ & $\begin{array}{l}6927 \\
(33.12)\end{array}$ & $\begin{array}{l}7244 \\
(32.92)\end{array}$ & $\begin{array}{l}7986 \\
(32.69)\end{array}$ & $\begin{array}{l}7762 \\
(33.2)\end{array}$ & $\begin{array}{l}8511 \\
(32.98)\end{array}$ & $\begin{array}{l}9006 \\
(32.57)\end{array}$ & $\begin{array}{l}8822 \\
(33.49)\end{array}$ & $\begin{array}{l}74299 \\
(33.21)\end{array}$ \\
\hline Dementia, $\mathrm{n}(\%)^{*}$ & $\begin{array}{l}1676 \\
(10.37)\end{array}$ & $\begin{array}{l}1973 \\
(9.98)\end{array}$ & $\begin{array}{l}1881 \\
(10.89)\end{array}$ & $\begin{array}{l}2062 \\
(9.86)\end{array}$ & $\begin{array}{l}2404 \\
(10.93)\end{array}$ & $\begin{array}{l}2672 \\
(10.94)\end{array}$ & $\begin{array}{l}2762 \\
(11.82)\end{array}$ & $\begin{array}{l}3182 \\
(12.33)\end{array}$ & $\begin{array}{l}3403 \\
(12.31)\end{array}$ & $\begin{array}{l}3062 \\
(11.62)\end{array}$ & $\begin{array}{l}25077 \\
(11.21)\end{array}$ \\
\hline Renal disease, $\mathrm{n}(\%)^{\star}$ & $\begin{array}{l}1893 \\
(11.71)\end{array}$ & $\begin{array}{l}2260 \\
(11.43)\end{array}$ & $\begin{array}{l}2187 \\
(12.67)\end{array}$ & $\begin{array}{l}2659 \\
(12.71)\end{array}$ & $\begin{array}{l}3082 \\
(14.01)\end{array}$ & $\begin{array}{l}3640 \\
(14.9)\end{array}$ & $\begin{array}{l}3878 \\
(16.59)\end{array}$ & $\begin{array}{l}4504 \\
(17.45)\end{array}$ & $\begin{array}{l}5160 \\
(18.66)\end{array}$ & $\begin{array}{l}5261 \\
(19.97)\end{array}$ & $\begin{array}{l}34524 \\
(15.43)\end{array}$ \\
\hline Any type of malignancy, $n(\%)^{*}$ & $\begin{array}{l}1154 \\
(7.14)\end{array}$ & $\begin{array}{l}1335 \\
(6.75)\end{array}$ & $\begin{array}{l}1323 \\
(7.66)\end{array}$ & $\begin{array}{l}1594 \\
(7.62)\end{array}$ & $\begin{array}{l}1634 \\
(7.43)\end{array}$ & $\begin{array}{l}1906 \\
(7.8)\end{array}$ & $\begin{array}{l}1953 \\
(8.35)\end{array}$ & $\begin{array}{l}2181 \\
(8.45)\end{array}$ & $\begin{array}{l}2350 \\
(8.5)\end{array}$ & $\begin{array}{l}2338 \\
(8.88)\end{array}$ & $\begin{array}{l}17768 \\
(7.94)\end{array}$ \\
\hline Any liver disease, $\mathrm{n}(\%)$ & $\begin{array}{l}793 \\
(4.91)\end{array}$ & $\begin{array}{l}1001 \\
(5.06)\end{array}$ & $\begin{array}{l}879 \\
(5.09)\end{array}$ & $\begin{array}{l}1059 \\
(5.06)\end{array}$ & $\begin{array}{l}1058 \\
(4.81)\end{array}$ & $\begin{array}{l}1248 \\
(5.11)\end{array}$ & $\begin{array}{l}1156 \\
(4.95)\end{array}$ & $\begin{array}{l}1220 \\
(4.73)\end{array}$ & $\begin{array}{l}1359 \\
(4.91)\end{array}$ & $\begin{array}{l}1407 \\
(5.34)\end{array}$ & $\begin{array}{l}11180 \\
(5)\end{array}$ \\
\hline Obesity, n (\%)* & $\begin{array}{l}1240 \\
(7.67)\end{array}$ & $\begin{array}{l}1593 \\
(8.06)\end{array}$ & $\begin{array}{l}1400 \\
(8.11)\end{array}$ & $\begin{array}{l}1766 \\
(8.44)\end{array}$ & $\begin{array}{l}1754 \\
(7.97)\end{array}$ & $\begin{array}{l}2339 \\
(9.58)\end{array}$ & $\begin{array}{l}2167 \\
(9.27)\end{array}$ & $\begin{array}{l}2599 \\
(10.07)\end{array}$ & $\begin{array}{l}2671 \\
(9.66)\end{array}$ & $\begin{array}{l}2822 \\
(10.71)\end{array}$ & $\begin{array}{l}20351 \\
(9.1)\end{array}$ \\
\hline Pleuritis, $n(\%) \dagger$ & $\begin{array}{l}913 \\
(5.65)\end{array}$ & $\begin{array}{l}1263 \\
(6.39)\end{array}$ & $\begin{array}{l}1107 \\
(6.41)\end{array}$ & $\begin{array}{l}1299 \\
(6.21)\end{array}$ & $\begin{array}{l}1391 \\
(6.32)\end{array}$ & $\begin{array}{l}1393 \\
(5.7)\end{array}$ & $\begin{array}{l}1326 \\
(5.67)\end{array}$ & $\begin{array}{l}1511 \\
(5.86)\end{array}$ & $\begin{array}{l}1611 \\
(5.83)\end{array}$ & $\begin{array}{l}1481 \\
(5.62)\end{array}$ & $\begin{array}{l}13295 \\
(5.94)\end{array}$ \\
\hline $\mathrm{CCl} 0, \mathrm{n}(\%) \dagger$ & $\begin{array}{l}4646 \\
(28.75)\end{array}$ & $\begin{array}{l}5726 \\
(28.97)\end{array}$ & $\begin{array}{l}4905 \\
(28.41)\end{array}$ & $\begin{array}{l}6175 \\
(29.53)\end{array}$ & $\begin{array}{l}6162 \\
(28.01)\end{array}$ & $\begin{array}{l}6782 \\
(27.77)\end{array}$ & $\begin{array}{l}6064 \\
(25.94)\end{array}$ & $\begin{array}{l}6429 \\
(24.91)\end{array}$ & $\begin{array}{l}6835 \\
(24.72)\end{array}$ & $\begin{array}{l}6237 \\
(23.68)\end{array}$ & $\begin{array}{l}59961 \\
(26.8)\end{array}$ \\
\hline $\mathrm{CCl} 1, \mathrm{n}(\%)^{*}$ & $\begin{array}{l}8140 \\
(50.37)\end{array}$ & $\begin{array}{l}9870 \\
(49.94)\end{array}$ & $\begin{array}{l}8663 \\
(50.17)\end{array}$ & $\begin{array}{l}10253 \\
(49.03)\end{array}$ & $\begin{array}{l}10951 \\
(49.77)\end{array}$ & $\begin{array}{l}12304 \\
(50.37)\end{array}$ & $\begin{array}{l}11865 \\
(50.76)\end{array}$ & $\begin{array}{l}13141 \\
(50.92)\end{array}$ & $\begin{array}{l}13929 \\
(50.37)\end{array}$ & $\begin{array}{l}13368 \\
(50.75)\end{array}$ & $\begin{array}{l}112484 \\
(50.28)\end{array}$ \\
\hline
\end{tabular}


Table 1 Continued

\begin{tabular}{|c|c|c|c|c|c|c|c|c|c|c|c|}
\hline & 2004 & 2005 & 2006 & 2007 & 2008 & 2009 & 2010 & 2011 & 2012 & 2013 & Total \\
\hline $\mathrm{CCl} \geq 2, \mathrm{n}(\%)^{*}$ & $\begin{array}{l}3375 \\
(20.88)\end{array}$ & $\begin{array}{l}4168 \\
(21.09)\end{array}$ & $\begin{array}{l}3699 \\
(21.42)\end{array}$ & $\begin{array}{l}4485 \\
(21.45)\end{array}$ & $\begin{array}{l}4889 \\
(22.22)\end{array}$ & $\begin{array}{l}5340 \\
(21.86)\end{array}$ & $\begin{array}{l}5448 \\
(23.3)\end{array}$ & $\begin{array}{l}6237 \\
(24.17)\end{array}$ & $\begin{array}{l}6891 \\
(24.92)\end{array}$ & $\begin{array}{l}6738 \\
(25.58)\end{array}$ & $\begin{array}{l}51270 \\
(22.92)\end{array}$ \\
\hline CAT, n (\%)* & $\begin{array}{l}1441 \\
(8.92)\end{array}$ & $\begin{array}{l}1759 \\
(8.9)\end{array}$ & $\begin{array}{l}1701 \\
(9.85)\end{array}$ & $\begin{array}{l}2229 \\
(10.66)\end{array}$ & $\begin{array}{l}2447 \\
(11.12)\end{array}$ & $\begin{array}{l}2656 \\
(10.87)\end{array}$ & $\begin{array}{l}2725 \\
(11.66)\end{array}$ & $\begin{array}{l}2929 \\
(11.35)\end{array}$ & $\begin{array}{l}3100 \\
(11.21)\end{array}$ & $\begin{array}{l}3071 \\
(11.66)\end{array}$ & $\begin{array}{l}24058 \\
(10.75)\end{array}$ \\
\hline Bronchial fibroscopy, $\mathrm{n}(\%) \dagger$ & $\begin{array}{l}424 \\
(2.62)\end{array}$ & $\begin{array}{l}516 \\
(2.61)\end{array}$ & $\begin{array}{l}418 \\
(2.42)\end{array}$ & $\begin{array}{l}521 \\
(2.49)\end{array}$ & $\begin{array}{l}540 \\
(2.45)\end{array}$ & $\begin{array}{l}583 \\
(2.39)\end{array}$ & $\begin{array}{l}537 \\
(2.3)\end{array}$ & $\begin{array}{l}564 \\
(2.19)\end{array}$ & $\begin{array}{l}581 \\
(2.1)\end{array}$ & $\begin{array}{l}642 \\
(2.44)\end{array}$ & $\begin{array}{l}5326 \\
(2.38)\end{array}$ \\
\hline Non-invasive MV, $n(\%)^{\star}$ & $\begin{array}{l}135 \\
(0.84)\end{array}$ & $\begin{array}{l}170 \\
(0.86)\end{array}$ & $\begin{array}{l}160 \\
(0.93)\end{array}$ & $\begin{array}{l}255 \\
(1.22)\end{array}$ & $\begin{array}{l}308 \\
(1.4)\end{array}$ & $\begin{array}{l}387 \\
(1.58)\end{array}$ & $\begin{array}{l}558 \\
(2.39)\end{array}$ & $\begin{array}{l}791 \\
(3.07)\end{array}$ & $\begin{array}{l}946 \\
(3.42)\end{array}$ & $\begin{array}{l}918 \\
(3.48)\end{array}$ & $\begin{array}{l}4628 \\
(2.07)\end{array}$ \\
\hline Invasive MV, n (\%)† & $\begin{array}{l}354 \\
(2.19)\end{array}$ & $\begin{array}{l}462 \\
(2.34)\end{array}$ & $\begin{array}{l}316 \\
(1.83)\end{array}$ & $\begin{array}{l}343 \\
(1.64)\end{array}$ & $\begin{array}{l}346 \\
(1.57)\end{array}$ & $\begin{array}{l}411 \\
(1.68)\end{array}$ & $\begin{array}{l}319 \\
(1.36)\end{array}$ & $\begin{array}{l}388 \\
(1.5)\end{array}$ & $\begin{array}{l}331 \\
(1.2)\end{array}$ & $\begin{array}{l}340 \\
(1.29)\end{array}$ & $\begin{array}{l}3610 \\
(1.61)\end{array}$ \\
\hline Thoracocentesis, $n(\%)^{*}$ & $\begin{array}{l}268 \\
(1.66)\end{array}$ & $\begin{array}{l}401 \\
(2.03)\end{array}$ & $\begin{array}{l}311 \\
(1.8)\end{array}$ & $\begin{array}{l}345 \\
(1.65)\end{array}$ & $\begin{array}{l}446 \\
(2.03)\end{array}$ & $\begin{array}{l}439 \\
(1.8)\end{array}$ & $\begin{array}{l}383 \\
(1.64)\end{array}$ & $\begin{array}{l}474 \\
(1.84)\end{array}$ & $\begin{array}{l}452 \\
(1.63)\end{array}$ & $\begin{array}{l}449 \\
(1.7)\end{array}$ & $\begin{array}{l}3968 \\
(1.77)\end{array}$ \\
\hline Red cell transfusion, $n(\%)^{*}$ & $\begin{array}{l}512 \\
(3.17)\end{array}$ & $\begin{array}{l}584 \\
(2.95)\end{array}$ & $\begin{array}{l}572 \\
(3.31)\end{array}$ & $\begin{array}{l}718 \\
(3.43)\end{array}$ & $\begin{array}{l}771 \\
(3.5)\end{array}$ & $\begin{array}{l}899 \\
(3.68)\end{array}$ & $\begin{array}{l}899 \\
(3.85)\end{array}$ & $\begin{array}{l}1054 \\
(4.08)\end{array}$ & $\begin{array}{l}1219 \\
(4.41)\end{array}$ & $\begin{array}{l}1051 \\
(3.99)\end{array}$ & $\begin{array}{l}8279 \\
(3.7)\end{array}$ \\
\hline Readmission, $\mathrm{n}(\%)^{*}$ & $\begin{array}{l}2031 \\
(12.57)\end{array}$ & $\begin{array}{l}2619 \\
(13.25)\end{array}$ & $\begin{array}{l}2267 \\
(13.13)\end{array}$ & $\begin{array}{l}2728 \\
(13.04)\end{array}$ & $\begin{array}{l}2948 \\
(13.4)\end{array}$ & $\begin{array}{l}3375 \\
(13.82)\end{array}$ & $\begin{array}{l}3274 \\
(14.01)\end{array}$ & $\begin{array}{l}3692 \\
(14.31)\end{array}$ & $\begin{array}{l}3971 \\
(14.36)\end{array}$ & $\begin{array}{l}3860 \\
(14.65)\end{array}$ & $\begin{array}{l}30765 \\
(13.75)\end{array}$ \\
\hline LOHS, median (IQR) & $8(5-13)$ & $8(5-13)$ & $8(5-13)$ & $8(5-12)$ & $8(5-12)$ & $8(5-12)$ & $8(5-12)$ & $7(5-11)$ & $7(5-11)$ & $7(4-11)$ & $8(5-12)$ \\
\hline IHM, n (\%)† & $\begin{array}{l}2232 \\
(13.81)\end{array}$ & $\begin{array}{l}2728 \\
(13.8)\end{array}$ & $\begin{array}{l}2345 \\
(13.58)\end{array}$ & $\begin{array}{l}2619 \\
(12.52)\end{array}$ & $\begin{array}{l}2797 \\
(12.71)\end{array}$ & $\begin{array}{l}3167 \\
(12.97)\end{array}$ & $\begin{array}{l}2987 \\
(12.78)\end{array}$ & $\begin{array}{l}3415 \\
(13.23)\end{array}$ & $\begin{array}{l}3728 \\
(13.48)\end{array}$ & $\begin{array}{l}3256 \\
(12.36)\end{array}$ & $\begin{array}{l}29274 \\
(13.09)\end{array}$ \\
\hline Streptococcus pneumoniae, $\mathrm{n}(\%) \dagger$ & $\begin{array}{l}2504 \\
(15.49)\end{array}$ & $\begin{array}{l}3411 \\
(17.26)\end{array}$ & $\begin{array}{l}2977 \\
(17.24)\end{array}$ & $\begin{array}{l}3873 \\
(18.52)\end{array}$ & $\begin{array}{l}3783 \\
(17.19)\end{array}$ & $\begin{array}{l}3992 \\
(16.34)\end{array}$ & $\begin{array}{l}3501 \\
(14.98)\end{array}$ & $\begin{array}{l}2380 \\
(9.22)\end{array}$ & $\begin{array}{l}2027 \\
(7.33)\end{array}$ & $\begin{array}{l}2095 \\
(7.95)\end{array}$ & $\begin{array}{l}30543 \\
(13.65)\end{array}$ \\
\hline Legionella, n (\%)† & $\begin{array}{l}154 \\
(0.95)\end{array}$ & $197(1)$ & $\begin{array}{l}207 \\
(1.2)\end{array}$ & $\begin{array}{l}189 \\
(0.9)\end{array}$ & $\begin{array}{l}217 \\
(0.99)\end{array}$ & $\begin{array}{l}213 \\
(0.87)\end{array}$ & $\begin{array}{l}197 \\
(0.84)\end{array}$ & $\begin{array}{l}158 \\
(0.61)\end{array}$ & $\begin{array}{l}170 \\
(0.61)\end{array}$ & $\begin{array}{l}159 \\
(0.6)\end{array}$ & $\begin{array}{l}1861 \\
(0.83)\end{array}$ \\
\hline Staphylococcus aureus, $\mathrm{n}(\%)^{*}$ & $\begin{array}{l}69 \\
(0.43)\end{array}$ & $\begin{array}{l}91 \\
(0.46)\end{array}$ & $\begin{array}{l}104 \\
(0.6)\end{array}$ & $\begin{array}{l}104 \\
(0.5)\end{array}$ & $\begin{array}{l}127 \\
(0.58)\end{array}$ & $\begin{array}{l}157 \\
(0.64)\end{array}$ & $\begin{array}{l}131 \\
(0.56)\end{array}$ & $\begin{array}{l}133 \\
(0.52)\end{array}$ & $\begin{array}{l}171 \\
(0.62)\end{array}$ & $\begin{array}{l}187 \\
(0.71)\end{array}$ & $\begin{array}{l}1274 \\
(0.57)\end{array}$ \\
\hline Haemophilus influenzae, n (\%) & $\begin{array}{l}58 \\
(0.36)\end{array}$ & $\begin{array}{l}63 \\
(0.32)\end{array}$ & $\begin{array}{l}57 \\
(0.33)\end{array}$ & $\begin{array}{l}85 \\
(0.41)\end{array}$ & $\begin{array}{l}77 \\
(0.35)\end{array}$ & $\begin{array}{l}76 \\
(0.31)\end{array}$ & $94(0.4)$ & $\begin{array}{l}81 \\
(0.31)\end{array}$ & $\begin{array}{l}92 \\
(0.33)\end{array}$ & $\begin{array}{l}109 \\
(0.41)\end{array}$ & $\begin{array}{l}792 \\
(0.35)\end{array}$ \\
\hline Pseudomonas aeruginosa, $\mathrm{n}(\%)$ & $\begin{array}{l}139 \\
(0.86)\end{array}$ & $\begin{array}{l}159 \\
(0.8)\end{array}$ & $\begin{array}{l}146 \\
(0.85)\end{array}$ & $\begin{array}{l}160 \\
(0.77)\end{array}$ & $\begin{array}{l}160 \\
(0.73)\end{array}$ & $\begin{array}{l}154 \\
(0.63)\end{array}$ & $\begin{array}{l}169 \\
(0.72)\end{array}$ & $\begin{array}{l}184 \\
(0.71)\end{array}$ & $\begin{array}{l}206 \\
(0.74)\end{array}$ & $\begin{array}{l}193 \\
(0.73)\end{array}$ & $\begin{array}{l}1670 \\
(0.75)\end{array}$ \\
\hline
\end{tabular}

Incidence was adjusted by age and sex.

${ }^{*} \mathrm{p}<0.05$ to assess increased time trend from 2004 to 2013

$t p<0.05$ to assess decreased time trend from 2004 to 2013.

AMI, acute myocardial infarction; CAT, computerised axial tomography of thorax; CCI, Charlson Comorbidity Index; CEVD/HP/PAPL, cerebrovascular disease/hemiplegia/paraplegia;

CHF, congestive heart failure; IHM, in-hospital mortality; LOHS, length of hospital stay; MV, mechanical ventilation; PVD, peripheral vascular disease. 
Table 2 Characteristics of hospital admissions for pneumonia as primary diagnosis in patients without type 2 diabetes in Spain, 2004-2013

\begin{tabular}{|c|c|c|c|c|c|c|c|c|c|c|c|}
\hline & 2004 & 2005 & 2006 & 2007 & 2008 & 2009 & 2010 & 2011 & 2012 & 2013 & Total \\
\hline $\mathrm{N}$ & 56991 & 66996 & 58233 & 66504 & 67123 & 74338 & 67643 & 72762 & 74816 & 72015 & 677421 \\
\hline Incidence* (per 100000 inhabitants) & 316.24 & 355.18 & 295.55 & 337.53 & 333.66 & 362.07 & 329.46 & 348.43 & 358.27 & 341.98 & 338.21 \\
\hline Women, $\mathrm{n}(\%)^{*}$ & $\begin{array}{l}20375 \\
(35.75)\end{array}$ & $\begin{array}{l}24955 \\
(37.25)\end{array}$ & $\begin{array}{l}21500 \\
(36.92)\end{array}$ & $\begin{array}{l}25263 \\
(37.99)\end{array}$ & $\begin{array}{l}26195 \\
(39.03)\end{array}$ & $\begin{array}{l}29742 \\
(40.01)\end{array}$ & $\begin{array}{l}26715 \\
(39.49)\end{array}$ & $\begin{array}{l}29407 \\
(40.42)\end{array}$ & $\begin{array}{l}30912 \\
(41.32)\end{array}$ & $\begin{array}{l}29455 \\
(40.9)\end{array}$ & $\begin{array}{l}264519 \\
(39.05)\end{array}$ \\
\hline Age, mean (SD) & $\begin{array}{l}73.96 \\
(13.25)\end{array}$ & $\begin{array}{l}74.26 \\
(13.36)\end{array}$ & $\begin{array}{l}74.24 \\
(13.64)\end{array}$ & $\begin{array}{l}74.33 \\
(13.78)\end{array}$ & $\begin{array}{l}74.79 \\
(13.78)\end{array}$ & $\begin{array}{l}73.77 \\
(14.61)\end{array}$ & $\begin{array}{l}75.67 \\
(13.72)\end{array}$ & $\begin{array}{l}75.65 \\
(13.86)\end{array}$ & $\begin{array}{l}77.02 \\
(13.28)\end{array}$ & $\begin{array}{l}76.36 \\
(13.73)\end{array}$ & $\begin{array}{l}75.06 \\
(13.76)\end{array}$ \\
\hline 40-64 years, $n(\%) \dagger$ & $\begin{array}{l}12471 \\
(21.88)\end{array}$ & $\begin{array}{l}14327 \\
(21.38)\end{array}$ & $\begin{array}{l}13036 \\
(22.39)\end{array}$ & $\begin{array}{l}15012 \\
(22.57)\end{array}$ & $\begin{array}{l}14768 \\
(22)\end{array}$ & $\begin{array}{l}18992 \\
(25.55)\end{array}$ & $\begin{array}{l}13930 \\
(20.59)\end{array}$ & $\begin{array}{l}15392 \\
(21.15)\end{array}$ & $\begin{array}{l}13497 \\
(18.04)\end{array}$ & $\begin{array}{l}14306 \\
(19.87)\end{array}$ & $\begin{array}{l}145731 \\
(21.51)\end{array}$ \\
\hline 65-74 years, $n(\%) \dagger$ & $\begin{array}{l}12578 \\
(22.07)\end{array}$ & $\begin{array}{l}14150 \\
(21.12)\end{array}$ & $\begin{array}{l}11555 \\
(19.84)\end{array}$ & $\begin{array}{l}12535 \\
(18.85)\end{array}$ & $\begin{array}{l}12153 \\
(18.11)\end{array}$ & $\begin{array}{l}12409 \\
(16.69)\end{array}$ & $\begin{array}{l}11068 \\
(16.36)\end{array}$ & $\begin{array}{l}11581 \\
(15.92)\end{array}$ & $\begin{array}{l}11321 \\
(15.13)\end{array}$ & $\begin{array}{l}10965 \\
(15.23)\end{array}$ & $\begin{array}{l}120315 \\
(17.76)\end{array}$ \\
\hline 75-84 years, $n(\%) \dagger$ & $\begin{array}{l}19395 \\
(34.03)\end{array}$ & $\begin{array}{l}23171 \\
(34.59)\end{array}$ & $\begin{array}{l}19755 \\
(33.92)\end{array}$ & $\begin{array}{l}22482 \\
(33.81)\end{array}$ & $\begin{array}{l}22213 \\
(33.09)\end{array}$ & $\begin{array}{l}23189 \\
(31.19)\end{array}$ & $\begin{array}{l}22555 \\
(33.34)\end{array}$ & $\begin{array}{l}23706 \\
(32.58)\end{array}$ & $\begin{array}{l}24640 \\
(32.93)\end{array}$ & $\begin{array}{l}23151 \\
(32.15)\end{array}$ & $\begin{array}{l}224257 \\
(33.1)\end{array}$ \\
\hline$\geq 85$ years, $n(\%)^{*}$ & $\begin{array}{l}12547 \\
(22.02)\end{array}$ & $\begin{array}{l}15348 \\
(22.91)\end{array}$ & $\begin{array}{l}13887 \\
(23.85)\end{array}$ & $\begin{array}{l}16475 \\
(24.77)\end{array}$ & $\begin{array}{l}17989 \\
(26.8)\end{array}$ & $\begin{array}{l}19748 \\
(26.57)\end{array}$ & $\begin{array}{l}20090 \\
(29.7)\end{array}$ & $\begin{array}{l}22083 \\
(30.35)\end{array}$ & $\begin{array}{l}25358 \\
(33.89)\end{array}$ & $\begin{array}{l}23593 \\
(32.76)\end{array}$ & $\begin{array}{l}187118 \\
(27.62)\end{array}$ \\
\hline AMI, n (\%) ${ }^{*}$ & $\begin{array}{l}1932 \\
(3.39)\end{array}$ & $\begin{array}{l}2309 \\
(3.45)\end{array}$ & $\begin{array}{l}1917 \\
(3.29)\end{array}$ & $\begin{array}{l}2279 \\
(3.43)\end{array}$ & $\begin{array}{l}2123 \\
(3.16)\end{array}$ & $\begin{array}{l}2207 \\
(2.97)\end{array}$ & $\begin{array}{l}1884 \\
(2.79)\end{array}$ & $\begin{array}{l}1955 \\
(2.69)\end{array}$ & $\begin{array}{l}1952 \\
(2.61)\end{array}$ & $\begin{array}{l}1802 \\
(2.5)\end{array}$ & $\begin{array}{l}20360 \\
(3.01)\end{array}$ \\
\hline $\mathrm{CHF}, \mathrm{n}(\%)^{*}$ & $\begin{array}{l}6649 \\
(11.67)\end{array}$ & $\begin{array}{l}7929 \\
(11.84)\end{array}$ & $\begin{array}{l}6761 \\
(11.61)\end{array}$ & $\begin{array}{l}8228 \\
(12.37)\end{array}$ & $\begin{array}{l}8673 \\
(12.92)\end{array}$ & $\begin{array}{l}9310 \\
(12.52)\end{array}$ & $\begin{array}{l}9516 \\
(14.07)\end{array}$ & $\begin{array}{l}10896 \\
(14.97)\end{array}$ & $\begin{array}{l}12004 \\
(16.04)\end{array}$ & $\begin{array}{l}11680 \\
(16.22)\end{array}$ & $\begin{array}{l}91646 \\
(13.53)\end{array}$ \\
\hline PVD, n (\%) ${ }^{*}$ & $\begin{array}{l}1857 \\
(3.26)\end{array}$ & $\begin{array}{l}2345 \\
(3.5)\end{array}$ & $\begin{array}{l}2121 \\
(3.64)\end{array}$ & $\begin{array}{l}2239 \\
(3.37)\end{array}$ & $\begin{array}{l}2237 \\
(3.33)\end{array}$ & $\begin{array}{l}2445 \\
(3.29)\end{array}$ & $\begin{array}{l}2496 \\
(3.69)\end{array}$ & $\begin{array}{l}2753 \\
(3.78)\end{array}$ & $\begin{array}{l}2923 \\
(3.91)\end{array}$ & $\begin{array}{l}3074 \\
(4.27)\end{array}$ & $\begin{array}{l}24490 \\
(3.62)\end{array}$ \\
\hline CEVD/HP/PAPL, n (\%)* & $\begin{array}{l}3993 \\
(7.01)\end{array}$ & $\begin{array}{l}4543 \\
(6.78)\end{array}$ & $\begin{array}{l}4020 \\
(6.9)\end{array}$ & $\begin{array}{l}4505 \\
(6.77)\end{array}$ & $\begin{array}{l}4969 \\
(7.4)\end{array}$ & $\begin{array}{l}5487 \\
(7.38)\end{array}$ & $\begin{array}{l}5561 \\
(8.22)\end{array}$ & $\begin{array}{l}6088 \\
(8.37)\end{array}$ & $\begin{array}{l}6508 \\
(8.7)\end{array}$ & $\begin{array}{l}6322 \\
(8.78)\end{array}$ & $\begin{array}{l}51996 \\
(7.68)\end{array}$ \\
\hline Chronic pulmonary disease, $n(\%) \dagger$ & $\begin{array}{l}20012 \\
(35.11)\end{array}$ & $\begin{array}{l}23065 \\
(34.43)\end{array}$ & $\begin{array}{l}19416 \\
(33.34)\end{array}$ & $\begin{array}{l}21961 \\
(33.02)\end{array}$ & $\begin{array}{l}21810 \\
(32.49)\end{array}$ & $\begin{array}{l}24120 \\
(32.45)\end{array}$ & $\begin{array}{l}22753 \\
(33.64)\end{array}$ & $\begin{array}{l}24112 \\
(33.14)\end{array}$ & $\begin{array}{l}24376 \\
(32.58)\end{array}$ & $\begin{array}{l}23789 \\
(33.03)\end{array}$ & $\begin{array}{l}225414 \\
(33.28)\end{array}$ \\
\hline Dementia, n (\%)* & $\begin{array}{l}5402 \\
(9.48)\end{array}$ & $\begin{array}{l}6295 \\
(9.4)\end{array}$ & $\begin{array}{l}5833 \\
(10.02)\end{array}$ & $\begin{array}{l}6224 \\
(9.36)\end{array}$ & $\begin{array}{l}6904 \\
(10.29)\end{array}$ & $\begin{array}{l}7599 \\
(10.22)\end{array}$ & $\begin{array}{l}7793 \\
(11.52)\end{array}$ & $\begin{array}{l}8536 \\
(11.73)\end{array}$ & $\begin{array}{l}9300 \\
(12.43)\end{array}$ & $\begin{array}{l}8742 \\
(12.14)\end{array}$ & $\begin{array}{l}72628 \\
(10.72)\end{array}$ \\
\hline Renal disease, $n(\%)^{\star}$ & $\begin{array}{l}4363 \\
(7.66)\end{array}$ & $\begin{array}{l}5327 \\
(7.95)\end{array}$ & $\begin{array}{l}4609 \\
(7.91)\end{array}$ & $\begin{array}{l}5760 \\
(8.66)\end{array}$ & $\begin{array}{l}6328 \\
(9.43)\end{array}$ & $\begin{array}{l}7189 \\
(9.67)\end{array}$ & $\begin{array}{l}7312 \\
(10.81)\end{array}$ & $\begin{array}{l}8278 \\
(11.38)\end{array}$ & $\begin{array}{l}9609 \\
(12.84)\end{array}$ & $\begin{array}{l}9572 \\
(13.29)\end{array}$ & $\begin{array}{l}68347 \\
(10.09)\end{array}$ \\
\hline Any type of malignancy, $n(\%)^{*}$ & $\begin{array}{l}5696 \\
(9.99)\end{array}$ & $\begin{array}{l}6420 \\
(9.58)\end{array}$ & $\begin{array}{l}6006 \\
(10.31)\end{array}$ & $\begin{array}{l}6671 \\
(10.03)\end{array}$ & $\begin{array}{l}6929 \\
(10.32)\end{array}$ & $\begin{array}{l}7642 \\
(10.28)\end{array}$ & $\begin{array}{l}7504 \\
(11.09)\end{array}$ & $\begin{array}{l}8234 \\
(11.32)\end{array}$ & $\begin{array}{l}8289 \\
(11.08)\end{array}$ & $\begin{array}{l}8407 \\
(11.67)\end{array}$ & $\begin{array}{l}71798 \\
(10.6)\end{array}$ \\
\hline Any liver disease, n (\%) & $\begin{array}{l}2825 \\
(4.96)\end{array}$ & $\begin{array}{l}3362 \\
(5.02)\end{array}$ & $\begin{array}{l}2942 \\
(5.05)\end{array}$ & $\begin{array}{l}3436 \\
(5.17)\end{array}$ & $\begin{array}{l}3409 \\
(5.08)\end{array}$ & $\begin{array}{l}3852 \\
(5.18)\end{array}$ & $\begin{array}{l}3457 \\
(5.11)\end{array}$ & $\begin{array}{l}3689 \\
(5.07)\end{array}$ & $\begin{array}{l}3733 \\
(4.99)\end{array}$ & $\begin{array}{l}3754 \\
(5.21)\end{array}$ & $\begin{array}{l}34459 \\
(5.09)\end{array}$ \\
\hline Obesity, n (\%)* & $\begin{array}{l}1940 \\
(3.4)\end{array}$ & $\begin{array}{l}2352 \\
(3.51)\end{array}$ & $\begin{array}{l}2044 \\
(3.51)\end{array}$ & $\begin{array}{l}2304 \\
(3.46)\end{array}$ & $\begin{array}{l}2489 \\
(3.71)\end{array}$ & $\begin{array}{l}3188 \\
(4.29)\end{array}$ & $\begin{array}{l}2839 \\
(4.2)\end{array}$ & $\begin{array}{l}3367 \\
(4.63)\end{array}$ & $\begin{array}{l}3393 \\
(4.54)\end{array}$ & $\begin{array}{l}3529 \\
(4.9)\end{array}$ & $\begin{array}{l}27445 \\
(4.05)\end{array}$ \\
\hline Pleuritis, n (\%)* & $\begin{array}{l}3992 \\
(7)\end{array}$ & $\begin{array}{l}4898 \\
(7.31)\end{array}$ & $\begin{array}{l}4240 \\
(7.28)\end{array}$ & $\begin{array}{l}4936 \\
(7.42)\end{array}$ & $\begin{array}{l}4760 \\
(7.09)\end{array}$ & $\begin{array}{l}4892 \\
(6.58)\end{array}$ & $\begin{array}{l}4782 \\
(7.07)\end{array}$ & $\begin{array}{l}4954 \\
(6.81)\end{array}$ & $\begin{array}{l}5054 \\
(6.76)\end{array}$ & $\begin{array}{l}5101 \\
(7.08)\end{array}$ & $\begin{array}{l}47609 \\
(7.03)\end{array}$ \\
\hline $\mathrm{CCl} 0, \mathrm{n}(\%) \dagger$ & $\begin{array}{l}18620 \\
(32.67)\end{array}$ & $\begin{array}{l}22311 \\
(33.3)\end{array}$ & $\begin{array}{l}19452 \\
(33.4)\end{array}$ & $\begin{array}{l}22643 \\
(34.05)\end{array}$ & $\begin{array}{l}21991 \\
(32.76)\end{array}$ & $\begin{array}{l}24786 \\
(33.34)\end{array}$ & $\begin{array}{l}20436 \\
(30.21)\end{array}$ & $\begin{array}{l}21691 \\
(29.81)\end{array}$ & $\begin{array}{l}21653 \\
(28.94)\end{array}$ & $\begin{array}{l}20550 \\
(28.54)\end{array}$ & $\begin{array}{l}214133 \\
(31.61)\end{array}$ \\
\hline $\mathrm{CCl} 1, \mathrm{n}(\%)^{*}$ & $\begin{array}{l}28219 \\
(49.51)\end{array}$ & $\begin{array}{l}32726 \\
(48.85)\end{array}$ & $\begin{array}{l}28327 \\
(48.64)\end{array}$ & $\begin{array}{l}31765 \\
(47.76)\end{array}$ & $\begin{array}{l}32752 \\
(48.79)\end{array}$ & $\begin{array}{l}35890 \\
(48.28)\end{array}$ & $\begin{array}{l}33399 \\
(49.38)\end{array}$ & $\begin{array}{l}36155 \\
(49.69)\end{array}$ & $\begin{array}{l}37205 \\
(49.73)\end{array}$ & $\begin{array}{l}35850 \\
(49.78)\end{array}$ & $\begin{array}{l}332288 \\
(49.05)\end{array}$ \\
\hline
\end{tabular}


Table 2 Continued

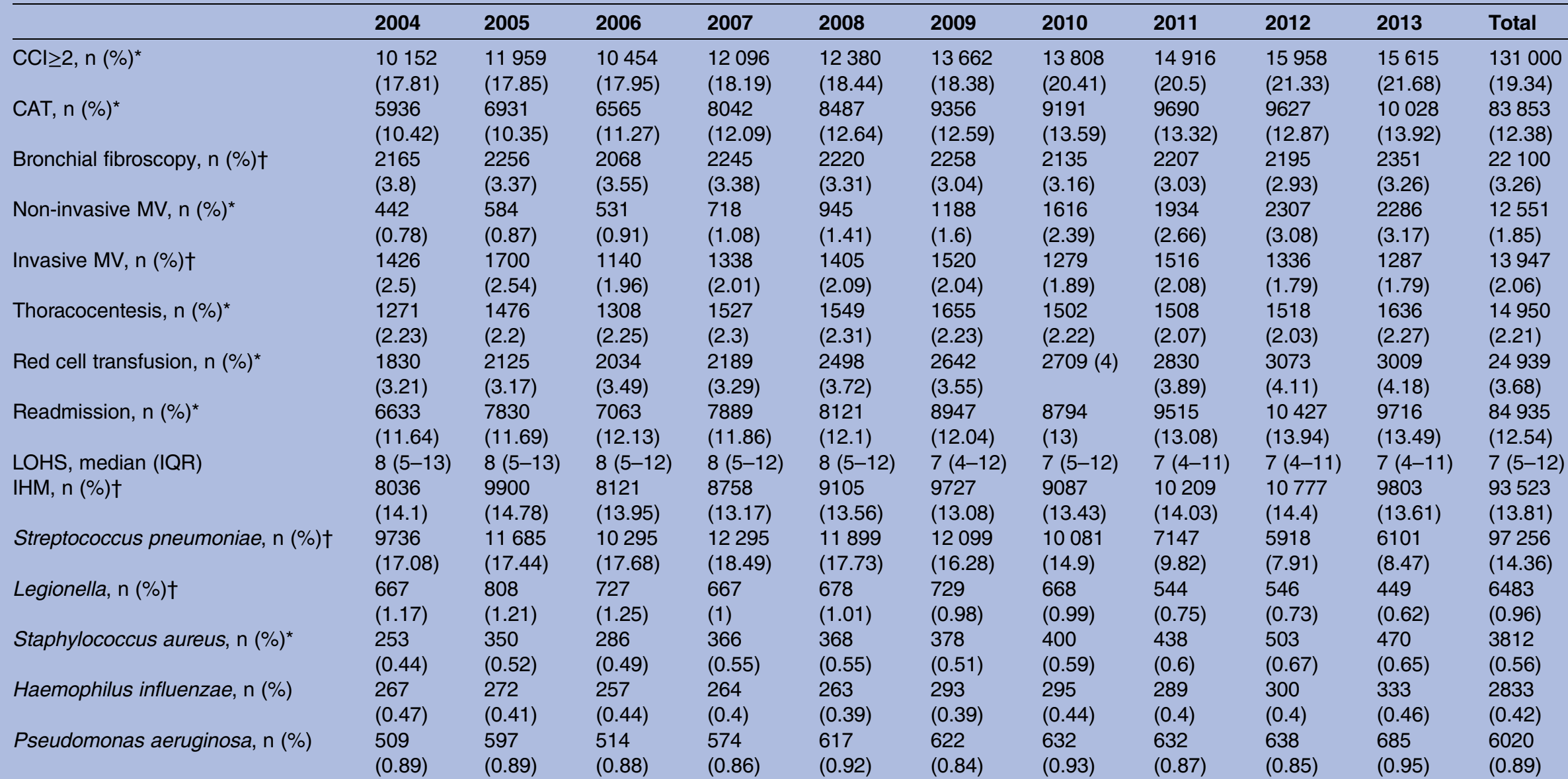

Incidence was adjusted by age and sex.

${ }^{*} p<0.05$ to assess increased time trend from 2004 to 2013.

$\dagger \mathrm{p}<0.05$ to assess decreased time trend from 2004 to 2013.

AMI, acute myocardial infarction; CAT, computerised axial tomography of thorax; CCI, Charlson Comorbidity Index; CEVD/HP/PAPL, cerebrovascular disease/hemiplegia/paraplegia;

CHF, congestive heart failure; IHM, in-hospital mortality; LOHS, length of hospital stay; MV, mechanical ventilation; PVD, peripheral vascular disease. 
Table 3 Characteristics of hospital admissions for pneumonia as primary diagnosis in patients with and without type 2 diabetes in Spain, 2001-2013, according to in-hospital mortality

\begin{tabular}{|c|c|c|c|c|}
\hline & \multicolumn{2}{|l|}{ Diabetes } & \multicolumn{2}{|l|}{ No diabetes } \\
\hline & Alive & Died & Alive & Died \\
\hline Women, n (\%)* $\dagger$ & 76301 (3924) & $12880(44)$ & $225626(3864)$ & 38893 (4159) \\
\hline Age, mean (SD) ${ }^{*} \dagger$ & $76.42(10.51)$ & $81.48(8.94)$ & $74.11(13.87)$ & $80.97(11.43)$ \\
\hline $40-64$ years, $n(\%)^{*} \dagger$ & 26507 (13.63) & $1489(5.09)$ & 136594 (23.39) & $9137(9.77)$ \\
\hline $65-74$ years, $n(\%)^{*} \dagger$ & $45070(23.18)$ & 3831 (13.09) & 109241 (18.71) & $11074(11.84)$ \\
\hline $75-84$ years, $n(\%)^{*} \dagger$ & 78684 (40.47) & $12132(41.44)$ & 192709 (33) & 31548 (33.73) \\
\hline$\geq 85$ years, $n(\%)^{\star} \dagger$ & 44180 (22.72) & $11822(40.38)$ & 145354 (24.89) & $41764(44.66)$ \\
\hline AMI, n $(\%)^{*} \dagger$ & $9163(4.71)$ & $1584(5.41)$ & $17000(2.91)$ & 3360 (3.59) \\
\hline $\mathrm{CHF}, \mathrm{n}(\%)^{*} \dagger$ & 35096 (18.05) & $6384(21.81)$ & 74122 (12.69) & $17524(18.74)$ \\
\hline PVD, n $(\%)^{*} \dagger$ & $11802(6.07)$ & $1927(6.58)$ & 20931 (3.58) & 3559 (3.81) \\
\hline CEVD/HP/PAPL, n (\%) ${ }^{\star} \dagger$ & $18199(9.36)$ & 4836 (16.52) & 40554 (6.95) & $11442(12.23)$ \\
\hline Chronic pulmonary disease, $\mathrm{n}(\%)^{\star} \dagger$ & $67498(34.71)$ & $6801(23.23)$ & 203108 (34.78) & $22306(23.85)$ \\
\hline Dementia, $\mathrm{n}(\%)^{\star} \dagger$ & $18657(9.6)$ & $6420(21.93)$ & $53446(9.15)$ & $19182(20.51)$ \\
\hline Renal disease, $n(\%)^{\star} \dagger$ & $29173(15)$ & $5351(18.28)$ & $55766(9.55)$ & $12581(13.45)$ \\
\hline Any type of malignancy, $n(\%)^{*} \dagger$ & $14089(7.25)$ & 3679 (12.57) & 56195 (9.62) & $15603(16.68)$ \\
\hline Any liver disease, $n(\%)$ & $9730(5)$ & $1450(4.95)$ & $29671(5.08)$ & $4788(5.12)$ \\
\hline Obesity, n (\%)* $\dagger$ & $19076(9.81)$ & $1275(4.36)$ & $25570(4.38)$ & $1875(2)$ \\
\hline Pleuritis, $n(\%)^{*} \dagger$ & $11774(6.06)$ & $1521(5.2)$ & $42000(7.19)$ & $5609(6)$ \\
\hline $\mathrm{CCl} 0, \mathrm{n}(\%)^{\star} \dagger$ & $54116(27.83)$ & 5845 (19.97) & 191839 (32.85) & $22294(23.84)$ \\
\hline $\mathrm{CCl} 1, \mathrm{n}(\%)^{*} \dagger$ & 97047 (49.91) & $15437(52.73)$ & 283688 (48.59) & $48600(51.97)$ \\
\hline $\mathrm{CCl} \geq 2, \mathrm{n}(\%)^{*} \dagger$ & $43278(22.26)$ & $7992(27.3)$ & 108371 (18.56) & $22629(24.2)$ \\
\hline CAT, n $(\%)^{*} \dagger$ & $22383(11.51)$ & $1675(5.72)$ & 77695 (13.31) & $6158(6.58)$ \\
\hline Bronchial fibroscopy, $\mathrm{n}(\%)^{*} \dagger$ & $4859(2.5)$ & $467(1.6)$ & $20039(3.43)$ & $2061(2.2)$ \\
\hline Non-invasive $\mathrm{MV}, \mathrm{n}(\%)^{\star} \dagger$ & $3563(1.83)$ & $1065(3.64)$ & $9250(1.58)$ & $3301(3.53)$ \\
\hline Invasive $\mathrm{MV}, \mathrm{n}(\%)^{*} \dagger$ & $1937(1)$ & $1673(5.71)$ & $7248(1.24)$ & $6699(7.16)$ \\
\hline Thoracocentesis, $n(\%)^{\star} \dagger$ & $3610(1.86)$ & $358(1.22)$ & $13535(2.32)$ & $1415(1.51)$ \\
\hline Red cell transfusion, $\mathrm{n}(\%)^{*} \dagger$ & $6866(3.53)$ & $1413(4.83)$ & 19626 (3.36) & $5313(5.68)$ \\
\hline Readmission, $\mathrm{n}(\%)^{*} \dagger$ & $24306(12.5)$ & $6459(22.06)$ & $66353(11.36)$ & $18582(19.87)$ \\
\hline LOHS, median (IQR) ${ }^{*} \dagger$ & $8(5-12)$ & $6(2-12)$ & $8(5-12)$ & $6(2-13)$ \\
\hline Streptococcus pneumoniae, $\mathrm{n}(\%)^{\star} \dagger$ & $28086(14.44)$ & 2457 (8.39) & $89171(15.27)$ & $8085(8.64)$ \\
\hline Legionella, $\mathrm{n}(\%)^{\star} \dagger$ & $1766(0.91)$ & $95(0.32)$ & $6134(1.05)$ & $349(0.37)$ \\
\hline Staphylococcus aureus, $\mathrm{n}(\%)^{\star} \dagger$ & $1042(0.54)$ & $232(0.79)$ & $3017(0.52)$ & $795(0.85)$ \\
\hline Haemophilus influenzae, $\mathrm{n}(\%)^{\star} \dagger$ & $763(0.39)$ & $29(0.1)$ & $2704(0.46)$ & $129(0.14)$ \\
\hline Pseudomonas aeruginosa, $\mathrm{n}(\%)^{\star} \dagger$ & $1400(0.72)$ & $270(0.92)$ & $4934(0.85)$ & $1086(1.16)$ \\
\hline
\end{tabular}

$S$ pneumoniae and Legionella decreased over time in people with T2DM and without diabetes. However, we detected a significant increase of $S$ aureus in both groups over the study period (table 1 and table 2). The prevalence of pathogens analysed was similar in patients with and without the disease.

Readmissions increased in both groups during the study (table 1 and table 2). Among diabetic patients, the increase was from $12.57 \%$ in 2004 to $14.65 \%$ in 2013. Equivalent figures for subjects without diabetes were significantly lower $(11.64 \%$ and $13.49 \%)$.

Overall median LOHS was significantly higher in patients with T2DM (8 vs7 days). Over time, LOHS following CAP fell significantly in patients with T2DM and without diabetes.

IHM was $13.81 \%$ for patients with T2DM and $13.09 \%$ for non-diabetic people $(\mathrm{p}<0.05)$. Crude IHM decreased significantly over time in people with T2DM and without diabetes (from $13.81 \%$ and $14.1 \%$, respectively, in 2004 to $12.36 \%$ and $13.61 \%$ in 2013 ), as can been seen in table 1 and table 2 .

Table 3 shows the characteristics of hospital admissions for CAP in patients with and without T2DM according to IHM during the study period.

For the entire time period, IHM was slightly but significantly higher among those without diabetes $(13.81 \%$ vs $13.09 \%)$.

Overall, patients with T2DM who died during their hospitalisation were significantly older (81.48; $\mathrm{SD}=8.94$ years) than those that survived (76.42; $\mathrm{SD}=10.51$ years) and had more coexisting medical conditions. Including higher prevalence of acute myocardial infarction $(5.41 \%$ vs $4.71 \%)$, congestive heart failure (21.81\% vs $18.05 \%)$, vascular disease $(6.58 \%$ vs, $6.07 \%)$, 
Table 4 Multivariate analysis of the factors potentially associated with in-hospital mortality for patients with and without type 2 diabetes in Spain, 2001-2013, with pneumonia as primary diagnosis

\begin{tabular}{|c|c|c|c|}
\hline & Diabetes OR $(95 \% \mathrm{Cl})$ & No diabetes OR $(95 \% \mathrm{Cl})$ & Total OR $(95 \% \mathrm{Cl})$ \\
\hline \multicolumn{4}{|l|}{ Age, years } \\
\hline $40-64$ & 1 & 1 & 1 \\
\hline $65-74$ & $1.47(1.38$ to 1.57$)$ & $1.47(1.42$ to 1.51$)$ & $1.46(1.42$ to 1.50$)$ \\
\hline $75-84$ & 2.70 (2.55 to 2.87$)$ & 2.49 (2.42 to 2.55$)$ & 2.53 (2.47 to 2.59$)$ \\
\hline$\geq 85$ & 4.75 (4.47 to 5.05$)$ & 4.52 (4.40 to 4.64$)$ & 4.55 (4.44 to 4.66$)$ \\
\hline \multicolumn{4}{|l|}{$\mathrm{CCl}$} \\
\hline 0 & 1 & 1 & 1 \\
\hline 1 & 1.35 (1.30 to 1.39$)$ & $1.28(1.26$ to 1.31$)$ & 1.30 (1.28 to 1.32$)$ \\
\hline$\geq 2$ & $1.50(1.44$ to 1.56$)$ & $1.44(1.41$ to 1.47$)$ & $1.46(1.43$ to 1.48$)$ \\
\hline Obesity & $0.51(0.48$ to 0.54$)$ & $0.50(0.47$ to 0.52$)$ & 0.50 (0.48 to 0.52$)$ \\
\hline Non-invasive MV & 2.04 (1.89 to 2.21$)$ & 2.01 (1.92 to 2.11$)$ & 2.02 (1.94 to 2.10$)$ \\
\hline Invasive MV & $11.53(10.68$ to 12.45$)$ & 12.55 (12.06 to 13.06$)$ & $12.34(11.91$ to 12.78$)$ \\
\hline Red cell transfusion & $1.14(1.07$ to 1.21$)$ & 1.35 (1.31 to 1.40$)$ & $1.30(1.26$ to 1.34$)$ \\
\hline Readmission & 1.91 (1.85 to 1.97$)$ & 1.85 (1.82 to 1.89$)$ & 1.87 (1.84 to 1.90$)$ \\
\hline CAT & $0.54(0.51$ to 0.57$)$ & $0.53(0.51$ to 0.55$)$ & 0.53 (0.52 to 0.55$)$ \\
\hline Thoracocentesis & $0.82(0.73$ to 0.93$)$ & $0.86(0.80$ to 0.91$)$ & $0.85(0.80$ to 0.90$)$ \\
\hline Bronchial fibroscopy & 0.75 (0.67 to 0.83$)$ & $0.71(0.67$ to 0.75$)$ & $0.72(0.68$ to 0.75$)$ \\
\hline Streptococcus & $0.54(0.52$ to 0.57$)$ & $0.52(0.51$ to 0.53$)$ & $0.52(0.51$ to 0.54$)$ \\
\hline \multicolumn{4}{|l|}{ pneumoniae* } \\
\hline Legionella* & $0.43(0.34$ to 0.53$)$ & 0.38 (0.34 to 0.42$)$ & $0.39(0.35$ to 0.43$)$ \\
\hline Staphylococcus & $1.22(1.04$ to 1.42$)$ & $1.26(1.16$ to 1.37$)$ & 1.25 (1.16 to 1.35$)$ \\
\hline \multicolumn{4}{|l|}{ aureus* } \\
\hline Haemophilus & $0.22(0.15$ to 0.32$)$ & $0.26(0.21$ to 0.31$)$ & $0.25(0.21$ to 0.29$)$ \\
\hline \multicolumn{4}{|l|}{ influenzae* } \\
\hline Year & $0.97(0.96$ to 0.99$)$ & $0.97(0.96$ to 0.98$)$ & $0.97(0.96$ to 0.98$)$ \\
\hline Diabetes & & - & $0.92(0.91$ to 0.94$)$ \\
\hline
\end{tabular}

cerebrovascular disease/hemiplegia/paraplegia $(16.52 \%$ vs $9.36 \%)$, dementia (21.93\% vs $9.6 \%)$, renal disease $(18.28 \%$ vs $15 \%)$ and any type of malignancy $(12.57 \%$ vs $7.25 \%)$. On the other hand, chronic obstructive pulmonary disease, obesity and pleuritis were more prevalent in diabetic patients that did not die during their hospital stay.

Invasive and non-invasive mechanical ventilation and red cell transfusion procedures were significantly more used in diabetic patients who died than in those that survived $(5.71 \%, 3.64 \%$ and $4.83 \%$ vs $1 \%, 1.83 \%$ and $3.53 \%$, respectively). However, CAT of thorax, thoracocentesis, bronchial fibroscopy were more frequent in T2DM and non-diabetic patients that survived than in those who died.

As can been seen in table 3, non-diabetic patients who died were significantly older, had more coexisting conditions like acute myocardial infarction, congestive heart failure, vascular disease, cerebrovascular disease/hemiplegia/paraplegia, dementia, renal disease and any type of malignancy, and underwent invasive and non-invasive mechanical ventilation and red cell transfusion procedures more than those non-diabetic patients that survived.

We found that $22.06 \%$ of diabetic patients that died and $12.5 \%$ of diabetic patients that survived were readmission $(\mathrm{p}<0.01)$. LOHS was 6 days in those diabetic and non-diabetic patients who died vs 8 days in those diabetic and non-diabetic patients that survived.

$S$ pneumoniae was more frequently detected in patients who lived than in those who died in T2DM and nondiabetic patients $(14.44 \%$ vs $8.39 \%$ and $15.27 \%$ vs $8.64 \%$ ), as can been seen in table 3 .

In table 4, we can see the results of the multivariate analysis of the factors independently associated with IHM in diabetic and non-diabetic patients during hospital admission for CAP in Spain for the period 2004-2013.

Among diabetic patients, IHM was significantly higher in older subjects (vs <40-64-year old, OR 4.75, 95\% CI 4.47 to 5.05 for $\geq 85$-year old) and in those with more comorbidities according to the CCI (vs no comorbidities, OR $1.35,95 \%$ CI 1.30 to 1.39 , for one comorbidity; OR $1.50,95 \%$ CI 1.44 to 1.56 , for two or more comorbidities).

For diabetic patients, IHM was significantly lower in obese persons (OR $0.51,95 \%$ CI 0.48 to 0.54 ) than in those with normal body mass index.

Over the entire study period, a diabetic patient with readmission was 1.14 (95\% CI 1.07 to 1.21 ) times more likely to die than a diabetic patient without readmission. 
Patients with T2DM having an in-hospital infection during admission for CAP ( $S$ pneumoniae or Legionella or $H$ influenzae were identified) had lower probability of dying than patients without these pathogens. However, diabetic patients with $S$ aureus had 1.22-fold higher probability of dying during their stay than those without that pathogen. IHM was significantly higher in patients who underwent invasive and non-invasive mechanical ventilation (OR 11.53, 95\% CI 10.68 to 12.45 and OR 2.04, $95 \%$ CI 1.89 to 2.21) and red cell transfusion (OR 1.14, $95 \%$ CI 1.07 to 1.21 ).

Diabetic patients who underwent CAT of thorax, bronchial fibroscopy and thoracocentesis procedures had a 0.54-fold, 0.75-fold and 0.82-fold, respectively, lower probability of dying during their stay than those who did not undergo these procedures.

Time trend analysis showed a minor but significant decrease in IHM from 2004 to 2013 in patients with T2DM (OR $0.97,95 \%$ CI 0.96 to 0.99 ).

As can been seen in table 4, for non-diabetic patients, IHM was significantly higher in older persons, in those with more comorbidities, in those with readmissions, in those with infections of $S$ aureus and in those who underwent invasive and non-invasive mechanical ventilation and red cell transfusion procedures. As for diabetic patients, we found a significant decrease in mortality over time.

In our study, suffering diabetes was associated with a lower IHM (OR $0.92,95 \%$ CI 0.91 to 0.94 ).

Finally, for the entire population, time trend analyses showed a significant decrease in mortality from 2004 to 2013 in patients admitted for CAP in Spain (OR 0.97, $95 \%$ CI 0.96 to 0.98$)$.

\section{DISCUSSION}

Using data from the CMBD, we found that rates of hospitalisation for CAP in patients with and without T2DM increased significantly from 2004 to 2013. These results are consistent with a report from Denmark, which pointed that total pneumonia hospitalisation increased by $63 \%$, from 4.96 per 1000 population in 1997 to 8.09 in 2011. ${ }^{12}$ Recently, Quan et al in Oxfordshire, UK, concluded that hospital admissions for CAP increased by 29\% per year between 2009 and $2014 .{ }^{9}$ The authors concluded that there was no evidence that the increase was caused by more low-severity cases presenting to hospital, $^{9}$ and that the ageing population only explains part of the increase. ${ }^{3} 917$

We found that readmissions for CAP increased over time in patients with and without T2DM and LOHS decreased in both groups of patients. These data are consistent with other published study, suggesting that the fact that readmissions for pneumonia increased over time supports another plausible explanation for the shortening of LOHS, namely, an increased pressure for early discharge. ${ }^{9} 18$

After adjusting for age and sex, we found that the incidence of CAP among patients with T2DM was 1.66-times higher than among non-diabetic patients. Our results agree with the Fremantle Diabetes Study data, in this study Hamilton et al compared patients with T2DM in Australia to matched non-diabetic subjects and indicated that IRR for pneumonia was 1.86 (95\% CI 1.55 to 2.21). ${ }^{6}$ In the USA, Jackson $e t a l^{19}$ also reported that the adjusted RR for hospitalisations for CAP was $1.52(95 \%$ CI 1.29 to 1.78 ) among patients with diabetes compared with patients without diabetes, based on 46237 subjects aged $>65$ years. In a Canadian study, the authors indicated that patients with diabetes had an increased risk of pneumonia-related hospitalisation than those without diabetes (RR $1.46,95 \%$ CI 1.42 to 1.49$).{ }^{8}$ In a casecontrol study in Denmark, Kornum et al found that T2DM was associated with a 1.2-fold increased risk of a pneumonia-related hospitalisation. They concluded that longer duration of diabetes and poor glycaemic control increase the risk of CAP-related hospitalisation.

Like other authors, we found that patients admitted for CAP were increasingly older over time. ${ }^{9}{ }^{17}$ In the UK, using linked electronic health records of patients with diabetes, McDonald et $a l^{20}$ observed that pneumonia incidence was $6-8$ times higher among patients aged $\geq 85$ years than patients aged $65-69$ years. Possible explanations include a general improvement in clinical management, especially changes in immunosuppressive regimens and handling of comorbidities. ${ }^{12}$

In our study, patients with T2DM had a higher number of simultaneous comorbidities and were more frequently obese, but obesity was not associated with a higher mortality risk during admission for CAP. Obesity is known to have adverse effects on immune function and to increase susceptibility to infections such as pneumonia; ${ }^{21}$ however, Hamilton et at concluded that a high body mass index was independently associated with any infection in their cohort of diabetic patients. A recent meta-analysis concluded that overweight and obesity were significantly associated with reduced risk of pneumonia mortality (RR $0.83,95 \%$ CI 0.77 to $0.91, \mathrm{p}<0.01$ ) and suggests that an 'obesity survival paradox' exists for pneumonia. ${ }^{22}$

The use of non-invasive mechanical ventilation has shown an over threefold increase in patients with and without T2DM over the study period. In a study about CAP in elderly, the authors found that mechanical ventilation was provided to $31.8 \%$ of patients and that almost half of the patients older than 90 years who received such care were discharged alive, supporting the belief that such care for the critically ill elderly patient is often justified. ${ }^{11}$ Our investigation showed that mechanical ventilation was a strong risk factor for IHM in both groups studied. However, given our study design it is not possible, with our data, to determine if mechanical ventilation is effective for critically ill elderly patient with CAP.

As expected, $S$ pneumoniae was the most frequent aetiological agent among patients with and without diabetes; however, its dominance is decreasing. Smith et al 
concluded that declines in cases of pneumonia due to $S$ pneumoniae (from $7.1 \%$ in 1993 to $2.3 \%$ 2011) may be related to more frequent and effective vaccination, which reduces the risk of invasive pneumococcal disease and bacteraemia. ${ }^{23}$ Also, this reduced risk may have resulted in less frequent coding because more thorough diagnostic evaluations accompany a higher severity of disease. In Spain $S$ pneumoniae vaccine is recommended for high-risk groups, including people with diabetes, and for all persons aged 65 years or over. ${ }^{24}$

We found that other organism's particularly $S$ aureus was more prevalent in dead patients than in survivors in patients with T2DM and non-diabetic patients. Like other authors, despite the trends observed, ${ }^{23}{ }^{25}$ the low incidence of $S$ aureus $(0.57 \%$ in patients with T2DM and $0.56 \%$ in those without T2DM), perhaps suggests that $S$ aureus is not routinely searched for and detected for patients with CAP. ${ }^{23}{ }^{26}$ It has been reported that pneumonia is the leading infectious cause of death in Spain; however, the mortality rate for pneumonia has decreased between 1980 and 2011. ${ }^{27}$ In our study, we found that crude IHM decreased over time among diabetic and non-diabetic patients with a diagnosis of CAP. Simonetti et $a l^{28}$ found a progressive downward trend of 30-day mortality in hospitalised patients with CAP $(-0.2 \%$ death/year; $p$ for trend $=0.003$ ) and concluded that the decreases in mortality rates suggest general improvement in the management of CAP.

We detected that patients with T2DM who died during their stay were older, had more coexisting comorbid conditions and had significantly more readmissions than those patients with T2DM that survived. In diabetic patients who died, mechanical ventilation and red cell transfusion were significantly more used than in those that survived. One possible explanation is that there is a trend to hospitalise a higher proportion of fragile or terminal patients who previously may have been treated at home. $^{12}$

In our population, the presence of T2DM was not a risk factor of death during admission for CAP. The results add important evidence to previous information. In an observational cohort study of all Medicare recipients, aged 65 years or older, hospitalised in non-federal US hospitals, Kaplan et $a l^{11}$ reported no association between IHM and diabetes. In a Canadian study of 2471 patients with CAP, the authors concluded that hyperglycaemia, not the presence of diabetes, was the only factor having a significant negative effect on patient survival. ${ }^{29}$ However, Kornum et a $\tilde{p}$ indicated that high glucose levels were associated with increased mortality in patients with and without T2DM. Perhaps, the fact is that patients with diabetes are more likely to be hospitalised with less severity. In fact, in our study, we observed a lower frequency of pleuritis and any type of malignancy in diabetics than in non-diabetics, which could justify the lower mortality in the first group. Finally, we think that this T2DM result is part of the obesity paradox. $^{22}$
In our study, mechanical ventilation (invasive and noninvasive) and red cell transfusion were significantly associated with mortality during admission for CAP in groups of patients with and without diabetes.

A recent study reported that non-invasive pressure ventilation is frequently used in CAP but is associated with high failure rates, and indicated that patients who failed non-invasive mechanical ventilation had an increased odds of death when compared with patients who were treated with invasive ventilation (OR 2.2, 95\% CI 1.0 to 4.8, $\mathrm{p}=0.03) .{ }^{30}$

The strengths of our findings lie in the large sample size, the 10-year follow-up period and the standardised methodology, which has been used to investigate diabetes and its complications in Spain and elsewhere. ${ }^{31}$

\section{Limitations of the study}

Nevertheless, our study is subject to several limitations. Our data source was the CMBD, an administrative database that contains discharge data for hospitalisations in Spain and uses information the physician has included in the discharge report. Therefore, our findings are limited by the lack of data, precluding adjustment for pneumococcal and influenza vaccinations, which have been associated with reduced mortality among patients hospitalised with pneumonia. ${ }^{5} \mathrm{~A}$ further limitation is the use of IHM which misses patients who may have died soon after discharge.

Other studies have identified factors that may influence in CAP outcomes and that were not included in our investigation because these variables were not collected in the CMBD. These factors include, among others, illness severity or antimicrobial treatments. ${ }^{32}$ Additionally, we also cannot identify whether gradual changes were made in referral practice during the study period.

Another significant limitation is the fact that we did not classify diabetic patients into groups based on the therapy used to control blood glucose, with the result that we were unable to provide data on the control of blood glucose during the hospitalisation.

The ICD-9-CM used in the CMBD does not contain any codes specifically for CAP but only has more general codes for pneumonia. Therefore, the ICD-9-CM cannot differentiate a CAP from a hospital-acquired pneumonia (HAP). In the CMBD database, the first diagnosis is the main reason why a patient is admitted to the hospital. By definition a patient with HAP has to acquire this infection after admission to the hospital. Therefore, according to this methodology, it is very improbable that an HAP could appear as a first diagnosis. The only possible situation for this would be that a patient previously hospitalised, and discharged from the hospital, would return in the first days with a pneumonia acquired in the previous hospitalisation. As commented before, we belief this is an extremely improbable situation that would only have a very small impact on the results. Furthermore, cases with a primary diagnosis of 
pneumonia (ICD-9-CM codes: 480-488, 507.0-507.8) in the hospital discharge report has been used by other authors, such as Kaplan et al and Hamilton et al, considering those as CAP admissions. ${ }^{6} 11$

Besides the limitations of administrative databases for clinical investigation on CAP, many studies have used these data sources for relevant epidemiological studies on respiratory diseases. ${ }^{6} 113334$ The CMBD is periodically audited and the validity of the 'diabetes diagnosis' in hospital discharge reports has been demonstrated in the past. ${ }^{35-38}$ However, as a result of these audits, it is possible and desirable that accuracy of coding may have improved over time so this would affect the results of our investigation and must be taken into consideration.

\section{CONCLUSIONS}

In conclusion, Spanish national data show that rates of hospitalisation for CAP in patients with and without T2DM increased significantly from 2004 to 2013 and incidence rates were higher in patients with T2DM than in those without diabetes in all time periods studied. CAP incidence seems to be increasing at a higher rate among patients with T2DM than among non-diabetic patients. IHM after CAP shows downward trend over time in all groups analysed. Remarkably, the presence of T2DM is not a risk factor of death after CAP in our cohort.

\section{Author affiliations}

${ }^{1}$ Preventive Medicine and Public Health Teaching and Research Unit, Health Sciences Faculty, Rey Juan Carlos University, Madrid, Spain

${ }^{2}$ Respiratory Care Department, Hospital General Universitario Gregorio Marañón, Universidad Complutense de Madrid, Madrid, Spain

${ }^{3}$ Internal Medicine Department, Hospital General Universitario Gregorio Marañón, Madrid, Spain

${ }^{4}$ Internal Medicine Department, Hospital Universitario Clínico San Carlos, Madrid, Spain

${ }^{5}$ Health Security Agency, Ministry of Health, Social Services and Equality, Madrid, Spain

${ }^{6}$ Dirección Técnica de Docencia e Investigación, Gerencia Atención Primaria, Madrid, Spain

Acknowledgements The authors would like to thank the Spanish Ministry of Health and Social Policy for providing the records of the Minimum Basic Data Set (MBDS).

Contributors $A L-d-A$ and RJ-G researched data, contributed to the discussion, wrote the manuscript, and reviewed/edited the manuscript. VH-B researched data and reviewed/edited the manuscript. JdM-D, IJ-T, JMdM-Y, MM-B, NP-F and MAnS-F contributed to the discussion and reviewed/edited the manuscript. All authors reviewed and gave their final approval of the version to be submitted.

Funding This study is a part of research funded by the FIS (Fondo de Investigaciones Sanitarias-Health Research Fund, grant number PI13/00118 and PI16/00 564, Instituto de Salud Carlos III) co-financed by the European Union through the Fondo Europeo de Desarrollo Regional (FEDER, 'Una manera de hacer Europa') and by the Grupo de Excelencia Investigadora URJC-Banco Santander N³0VCPIGI03: Investigación traslacional en el proceso de salud-enfermedad (ITPSE).

Competing interests None declared.

Ethics approval Ethics Committee of the Universidad Rey Juan Carlos.
Provenance and peer review Not commissioned; externally peer reviewed.

Data sharing statement No additional data are available.

Open Access This is an Open Access article distributed in accordance with the Creative Commons Attribution Non Commercial (CC BY-NC 4.0) license, which permits others to distribute, remix, adapt, build upon this work noncommercially, and license their derivative works on different terms, provided the original work is properly cited and the use is non-commercial. See: http:// creativecommons.org/licenses/by-nc/4.0/

\section{REFERENCES}

1. Soriguer F, Goday A, Bosch-Comas A, et al. Prevalence of diabetes mellitus and impaired glucose regulation in Spain: the Di@ bet.es Study. Diabetologia 2012;55:88-93.

2. Korbel L, Spencer JD. Diabetes mellitus and infection: an evaluation of hospital utilization and management costs in the United States. J Diabetes Complicat 2015;29:192-5.

3. Fry AM, Shay DK, Holman RC, et al. Trends in hospitalizations for pneumonia among persons aged 65 years or older in the United States, 1988-2002. JAMA 2005;294:2712-19.

4. Kornum JB, Thomsen RW, Riis A, et al. Diabetes, glycemic control, and risk of hospitalization with pneumonia. A population-based case-control study. Diabetes Care 2008;31:1541-5.

5. Kornum JB, Thomsen RW, Riis A, et al. Type 2 diabetes and pneumonia outcomes. A population-based cohort study. Diabetes Care 2007;30:2251-7.

6. Hamilton EJ, Martin N, Makepeace A, et al. Incidence and predictors of hospitalization for bacterial infection in community-based patients with type 2 diabetes: the fremantle diabetes study. PLOS ONE 2013;8:e60502.

7. Benfield T, Jensen JS, Nordestgaard BG. Influence of diabetes and hyperglycaemia on infectious disease hospitalisation and outcome. Diabetologia 2007;50:549-54.

8. Shah BR, Hux JE. Quantifying the risk of infectious diseases for people with diabetes. Diabetes Care 2003;26:510-13.

9. Quan TP, Fawcett NJ, Wrightson JM, et al. Increasing burden of community-acquired pneumonia leading to hospitalisation, 19982014. Thorax 2016;71:535-42.

10. Koziel H, Koziel MJ. Pulmonary complications of diabetes mellitus. Pneumonia. Infect Dis Clin North Am 1995;9:65-96.

11. Kaplan V, Angus DC, Griffin MF, et al. Hospitalized communityacquired pneumonia in the elderly: age- and sex-related patterns of care and outcome in the United States. Am J Respir Crit Care Med 2002;165:766-72.

12. Søgaard M, Nielsen RB, Schønheyder HC, et al. Nationwide trends in pneumonia hospitalization rates and mortality, Denmark 19972011. Respir Med 2014;108:1214-22.

13. Instituto Nacional de Gestión Sanitaria, Ministerio de Sanidad, Servicios Sociales e Igualdad. Conjunto Mínimo Básico de Datos, Hospitales del INSALUD. http://www.ingesa.msc.es/estadEstudios/ documPublica/CMBD-2001.htm (accessed 6 Oct 2015).

14. Charlson ME, Pompei P, Ales KL, et al. A new method of classifying prognostic comorbidity in longitudinal studies: development and validation. J Chronic Dis 1987;40:373-83.

15. Ministerio de Sanidad, Servicios Sociales e Igualdad. [Encuesta Nacional de Salud de España]. http://www.msssi.gob.es/ estadEstudios/estadisticas/encuestaNacional/ (accessed 1 Dec 2015).

16. Instituto Nacional de Estadística. Population estimates. 2010. http://www.ine.es (accessed 1 Dec 2015).

17. Jain S, Self WH, Wunderink RG, et al. Community-acquired pneumonia requiring hospitalization among U.S. Adults. N Engl J Med 2015;373:415-27.

18. Sicras-Mainar A, Ibáñez-Nolla J, Cifuentes I, et al. Retrospective epidemiological study for the characterization of communityacquired pneumonia and pneumococcal pneumonia in adults in a well-defined area of Badalona (Barcelona, Spain). BMC Infect Dis 2012;12:283.

19. Jackson ML, Neuzil KM, Thompson WW, et al. The burden of community-acquired pneumonia in seniors: results of a population-base study. Clin Infect Dis 2004;39:1642-50.

20. McDonald HI, Nitsch D, Millett ER, et al. New estimates of the burden of acute community-acquired infections among older people with diabetes mellitus: a retrospective cohort study using linked electronic health records. Diabet Med 2014;31:606-14. 
21. Mancuso P. Obesity and respiratory infections: does excess adiposity weigh down host defense? Pulm Pharmacol Ther 2013;26:412-19.

22. Nie $\mathrm{W}$, Zhang $\mathrm{Y}$, Jee $\mathrm{SH}$, et al. Obesity survival paradox in pneumonia: a meta-analysis. BMC Med 2014;12:61.

23. Smith SB, Ruhnke GW, Weiss $\mathrm{CH}$, et al. Trends in pathogens among patients hospitalized for pneumonia from 1993 to 2011. JAMA Intern Med 2014;174:1837-9.

24. Ministry of Health, Social Services and Equality. Vaccination against pneumococcal infections in risk groups. http://www.msssi.gob.es/ profesionales/saludPublica/prevPromocion/vacunaciones/docs/ Neumococo Gruposriesgo.pdf (accessed 3 Apr 2016).

25. Lewis SS, Walker VJ, Lee MS, et al. Epidemiology of methicillinresistant Staphylococcus aureus pneumonia in community hospitals. Infect Control Hosp Epidemiol 2014;35:1452-7.

26. Wunderink RG. Community-acquired pneumonia versus healthcare-associated pneumonia. The returning pendulum. Am J Respir Crit Care Med 2013;188:896-8.

27. López-Cuadrado T, Llácer A, Palmera-Suárez R, et al. Trends in infectious disease mortality rates, Spain, 1980-2011. Emerging Infect Dis 2014;20:782-9.

28. Simonetti AF, Garcia-Vidal C, Viasus D, et al. Declining mortality among hospitalized patients with community-acquired pneumonia. Clin Microbiol Infect 2016;22:567.e1-7.

29. McAlister FA, Majumdar SR, Blitz $S$, et al. The relation between hyperglycemia and outcomes in 2,471 patients admitted to the hospital with community-acquired pneumonia. Diabetes Care 2005;28:810-15.

30. Murad A, Li PZ, Dial S, et al. The role of noninvasive positive pressure ventilation in community-acquired pneumonia. J Crit Care 2015;30:49-54.
31. Jiménez-García R, Hernández-Barrera $\mathrm{V}$, Rodríguez-Rieiro $\mathrm{C}$, et al. Hospitalizations from pandemic Influenza [A(H1N1)pdm09] infections among type 1 and 2 diabetes patients in Spain. Influenza Other Respir Viruses 2013;7:439-47.

32. Luna CM, Palma I, Niederman MS, et al. The impact of age and comorbidities on the mortality of patients of different age groups admitted with community-acquired pneumonia. Ann Am Thorac Soc 2016;13:1519-26.

33. Sánchez-Muñoz G, López de Andrés A, Jiménez-García R, et al. Time trends in hospital admissions for bronchiectasis: analysis of the Spanish national hospital discharge data (2004 to 2013). PLOS ONE 2016;11:e0162282.

34. de Miguel-Díez J, Muñoz-Rivas N, Jiménez-García R, et al. Type 2 diabetes is associated with a higher incidence of hospitalization for pulmonary embolism in Spain: Analysis of hospital discharge data during 2004-2013. Respirology 2016;21:1277-84.

35. Ribera A, Marsal JR, Ferreira-González I, et al. Predicting in-hospita mortality with coronary bypass surgery using hospital discharge data: comparison with a prospective observational study. Rev Esp Cardiol 2008;61:843-52.

36. Leong A, Dasgupta K, Bernatsky S, et al. Systematic review and meta-analysis of validation studies on a diabetes case definition from health administrative records. PLOS ONE 2013;8: e75256.

37. Zellweger U, Bopp M, Holzer BM, et al. Prevalence of chronic medical conditions in Switzerland: exploring estimates validity by comparing complementary data sources. BMC Public Health 2014;14:1157.

38. Chen $\mathrm{G}$, Khan N, Walker R, et al. Validating ICD coding algorithms for diabetes mellitus from administrative data. Diabetes Res Clin Pract 2010;89:189-95. 